Cinémas

Revue d'études cinématographiques

Journal of Film Studies

\title{
Cinéma québécois à l'ombre de la mélancolie
}

\section{Heinz Weinmann}

Volume 8, numéro 1-2, automne 1997

Cinéma et mélancolie

URI : https://id.erudit.org/iderudit/024741ar

DOI : https://doi.org/10.7202/024741ar

Aller au sommaire du numéro

Éditeur(s)

Cinémas

ISSN

1181-6945 (imprimé)

1705-6500 (numérique)

Découvrir la revue

Citer cet article

Weinmann, H. (1997). Cinéma québécois à l’ombre de la mélancolie. Cinémas, 8(1-2), 34-46. https://doi.org/10.7202/024741ar

\section{Résumé de l'article}

Dans cet article, tout en s'appuyant sur la définition freudienne de la mélancolie, l'auteur cherche à analyser un corpus de trois oeuvres cinématographiques québécoises : La Femme de l'hôtel de Léa Pool (1984), Cargo de François Girard (1990) et Léolo de Jean-Claude Lauzon (1992), trois films qui s'inscrivent dans ce qu'on a appelé le « syndrome postréférendaire », provoqué par l'échec du premier référendum sur la souveraineté-association en 1980. d'utilisation que vous pouvez consulter en ligne.

https://apropos.erudit.org/fr/usagers/politique-dutilisation/ 


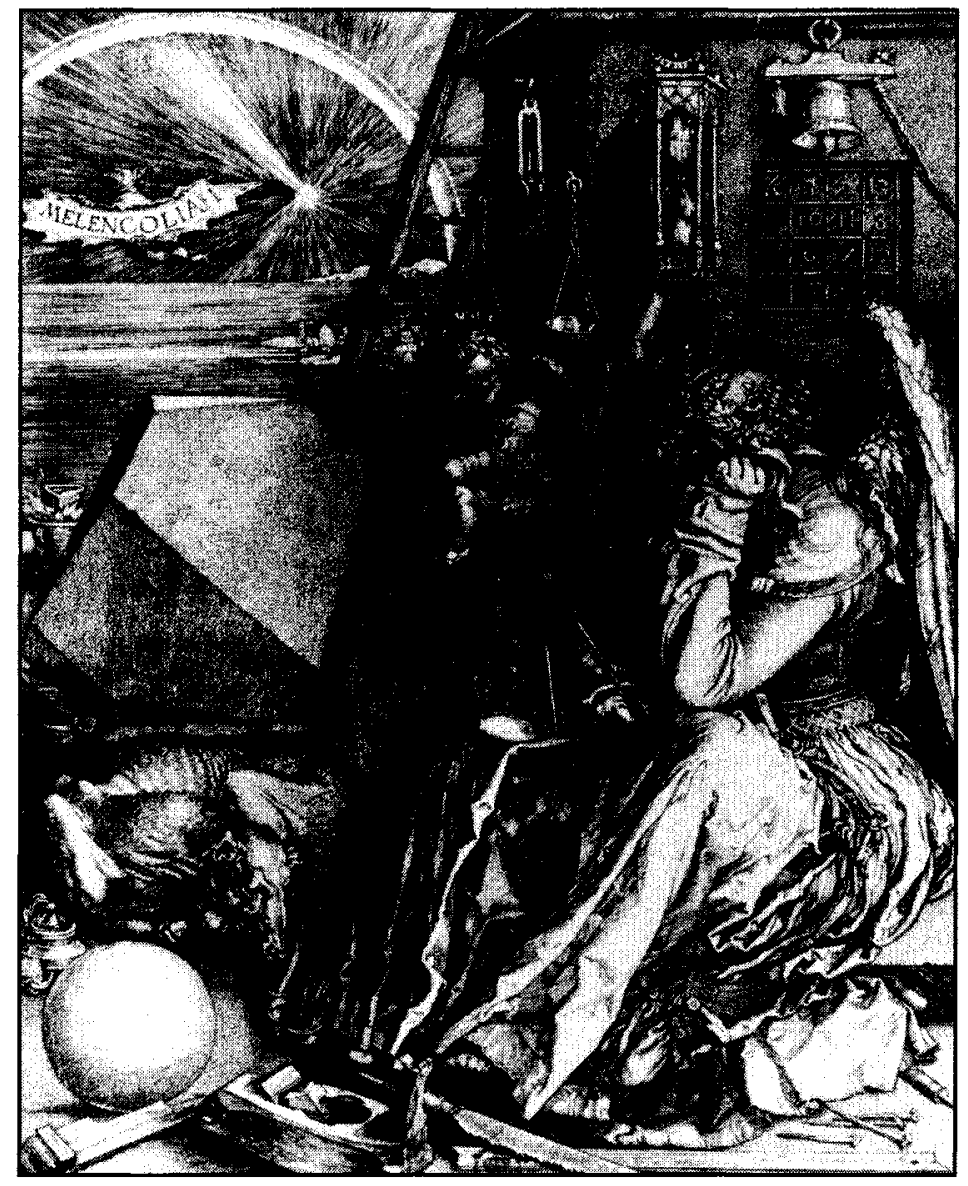

Dürer, Melencolia I, gravure 


\title{
Cinéma québécois à l’ombre de la mélancolie
}

\section{Heinz Weinmann}

\section{RÉSUMÉ}

Dans cet article, tout en s'appuyant sur la définition freudienne de la mélancolie, l'auteur cherche à analyser un corpus de trois œuvres cinématographiques québécoises: La Femme de l'hôtel de Léa Pool (1984), Cargo de François Girard (1990) et Léolo de Jean-Claude Lauzon (1992), trois films qui s'inscrivent dans ce qu'on a appelé le "syndrome postréférendaire", provoqué par l'échec du premier référendum sur la souveraineté-association en 1980.

\begin{abstract}
In this article, relying on the Freudian definition of melancholy, the author analyses a corpus of three Quebec films: La Femme de l'hôtel by Léa Pool (1984), Cargo by François Girard (1990), and Léolo by JeanClaude Lauzon (1992). What these three films share has been called the "post-referendum syndrome" that was brought on by the failure of the first referendum on sovereignty-association in 1980.
\end{abstract}

L'ombre de la mélancolie tombera aussi sur ce moi qui peut alors être jugé par une instance particulière comme un objet, comme un objet abandonné.

Sigmund Freud 
Notre art signifie un éblouissement devant la vérité. La lumière sur le masque qui recule est vrai, rien d'autre.

Franz Kafka

I

Comme l'a magistralement montré Saturne et la Mélancolie (Klibansky et al., 1989), depuis l'antiquité gréco-romaine, la mélancolie a gravité autour de deux pôles: d'un côté le pôle créateur, inauguré par Aristote, qui considère la mélancolie comme un des traits essentiels du "génie"; de l'autre, comme dans la célèbre Melencolia I de Dürer, le pôle de l'abattement humain, écrasé par une matière qu'il refuse de travailler. Or, au fur et à mesure que la clinique, au XIX ${ }^{c}$ siècle, s'accapare de la mélancolie, cette dernière se départit de sa dualité originelle pour se réduire à une pathologie dans l'ensemble d'une nosologie médicale.

Pour Freud, la psychanalyse naissante devait nécessairement passer par un détachement radical de la clinique ${ }^{1}$. Dans la foulée de cette titanesque transmutation, la psychanalyse redéfinira dans une perspective psychanalytique des notions cliniques telles que névrose, psychose et mélancolie. Entreprise tardivement, la redéfinition de la mélancolie, Freud l'opère en 1915, lors du vaste réaménagement "économique" de la psychanalyse, appelée "métapsychologie " et dont l'article "Deuil et mélancolie" fait partie.

Dans ce qui suit, tout en nous appuyant sur la définition freudienne de la mélancolie, nous cherchons à élucider un corpus de trois œuvres cinématographiques québécoises: La Femme de l'hôtel de Léa Pool (1984), Cargo de François Girard (1990), et Léolo de Jean-Claude Lauzon (1992). Plus nettement que les deux autres, le premier film s'inscrit dans la mouvance appelée "syndrome postréférendaire", provoqué suite à l'échec du premier référendum de 1980. Mais plus qu'une réponse ponctuelle à une situation politique décevante, nous voulons analyser dans les trois films plus globalement le rôle de la mélancolie dans la psyché québécoise en tant que pharmakon ${ }^{2}$, défense contre le danger de mort d'un idéal (souveraineté, indépendance). Ne voulant, ne pouvant faire le deuil de l'idéal en général, de l'idée 
de souveraineté en particulier, le Québécois préfere "payer» de sa personne en s'infligeant les affres de la mélancolie. Car il y a de l'espoir tant que l'idéal n'est pas mort...

Freud a établi un parallèle entre la mélancolie et le deuil : siil y a perte dans les deux cas, celle de l'endeuillé est irréversible, alors que celle du mélancolique, loin d'être totale, ne touche que l'affection de l'objet d'amour. Restant affectivement attaché à l'objet, le mélancolique cherche à attirer son attention par des voies / voix détournées, souvent contraires à celles ouvertement énoncées. Par toutes sortes de postures de l'appauvrissement (délire de petitesse), en faisant pitié, le mélancolique cherche à attirer l'attention de l'objet. "Dans le deuil le monde est devenu pauvre et vide, dans la mélancolie c'est le moi lui-même. Le malade nous dépeint son moi comme sans valeur, incapable de quoi que ce soit et moralement condamnable» (Freud, p. 152).

C'est ce vide, provoqué par la perte d'amour de l'objet d'amour, qui se trouve au centre de La Femme de l'hôtel de Léa Pool. Nous cherchons à cerner par quelles stratégies filmiques Léa Pool énonce ce vide. Elles sont de trois ordres: 1) direction de la caméra ; 2) déconnexion de l'espace-temps comme définitions humaines; 3 ) disparition des définitions ayant trait aux personnes. Analysons dans l'ordre le fonctionnement de ces trois stratégies dans La Femme de l'hôtel.

Dans le premier plan, la caméra balaie lentement, méthodiquement le centre-ville de Montréal. Contrairement à la pratique habituelle, ce mouvement ne s'arrête pas, ne fait pas de zoom sur une maison, ne reconnaissant aucun espace d'habitation privilégié. Tous les lieux s'équivalent dans leur insignifiance foncière, aucun ne vaut d'être singularisé.

Le travelling lent du début signifie que cette ville, Montréal, n'est pas un lieu d'habitation ${ }^{3}$ mais un lieu de passage, un espace nomade 4 . L'hôtel en est sa métaphore. Les personnages n'ont pas de chez-soi, pas de "propriété" - au double sens du terme - qui les définisse, qui les singularise. "Rien n'est à vous", note Estelle (Louise Marleau), «la femme de l'hôtel ". Le mouvement de balayage mécanique de la ville accentue la situation de désappropriation des personnages. Ils ont été spoliés de tout, même de leur propre passé. 
Andréane Richler (Paule Baillargeon), la cinéaste, d'entrée de jeu, prend des photos de la ville comme une touriste qui vient de découvrir Montréal. Tout en collant les photos dans un album, elle note: "Il m'a fallu tout redécouvrir et il m'a fallu oublier et recommencer. J'ai "parcouru” la ville de fond en comble.» Nous avons souligné le mot clé «parcourir» qui illustre bien l'errance, sans but précis, des personnages.

Dans ce film, la caméra ne se fait jamais la complice du regard humain. Elle enregistre plus qu'elle ne donne à voir. Lorsque la caméra cible le regard d'Estelle, revenue à l'hôtel après une rencontre - d'ailleurs laissée en blanc - avec Andréane, la caméra figure indirectement le vide de ce regard mélancolique qui ne voit rien. La conduite de la caméra suggère ainsi le pathos au sens premier de "souffrance" devant la perte de l'amour de l'objet.

Cet effet de la caméra crée pour ainsi dire un «inconscient filmique" du fait qu'il laisse en blanc, refoulé dans l'inconscient, l'amour pour cet objet. La caméra n'enregistre que les effets, les symptômes du mélancolique, laissant dans l'ombre ses causes cachées. La grande règle de la logique narrative occidentale du ante hoc propter hoc perd ici complètement sa raison d'être: chronos cesse de structurer logos. L'avant et l'après perdent leur rôle essentiel de lien causal. Nous avons vu que la libido du mélancolique, après la perte de l'amour de l'objet, reflue sur lui-même, se punissant pour ainsi dire de cette perte. Dans La Femme de l'hôtel, la tentative de suicide d'Estelle au moyen de barbituriques vient immédiatement après sa rencontre avec Andréane, mais rien n'indique au spectateur que cette dernière en a été la "cause".

Dans La Femme de l'hôtel, le temps et l'espace ont perdu leur rôle de marqueurs d'identification de l'individu. Puisqu'il vient juste d'être question du temps, concentrons-nous davantage sur l'espace. Tout comme l' "habitant", le nomade a son ancrage dans le passé du Canada français, dans le coureur des bois ${ }^{5}$, ce mal aimé de l'histoire québécoise, puisque son nomadisme invétéré jure avec l'affirmation de la territorialité de l'espace québécois. Pour ajouter à leur malheur, ces coureurs des bois canadiens-français ont découvert, exploré l'espace continental non seulement canadien mais américain. En montrant, dans son der- 
nier film Le Mouvement du désir (1993), le train filiforme qui fraie sa voie dans un espace qui défile à toute vitesse d'est en ouest, Léa Pool a rendu visible ce désir du mouvement inhérent à l'histoire du Canada français.

Or, le mouvement dans La Femme de l'hôtel est sans désir, les rapports changeants, aléatoires entre les personnages reflètent le nomadisme de nos cités modernes, avec comme conséquence la solitude de l'individu dans la foule, thème traité magistralement par Poe dans le conte L'homme des foules. Mouvement pour le mouvement, sans but, sans raison. Ainsi, le premier plan d'Estelle cadre ses pieds, ses jambes comme organes de locomotion humaine du reste de son corps, notamment de sa tête, pour ensuite se porter sur sa valise. Visiblement, cette femme se prépare à un voyage sans en connaître la destination ${ }^{6}$. Arrivée à la gare, devant le train en partance pour Vancouver, elle hésite, elle recule et retourne à l'hôtel. Sans but, les personnages restent prisonniers d'une errance, symbole de leur égarement, dont la chanteuse créée par Andréane (Paule Baillargeon), devenue "folle", internée, constitue le cas le plus extrême".

La cinéaste est bien consciente de l'espace nomade dans lequel elle inscrit sa "créature". "Une rue, un théâtre, une ville, ça ne ressemble jamais à ce qu'on a imaginé, encore moins aux souvenirs qu'on en a gardés." Ces lieux de passage fragmentés, dont l'hôtel devient le symbole, cessent d'être des espaces mémoriels par rapport auxquels les personnages se définissent. Lieu de déréliction, de solitude existentielle, l'hôtel creuse un fossé large et profond entre lui et son contraire, hors d'atteinte: le chez-soi, le foyer.

Enfin, ce film pose la question de l'identité des personnages. On est en droit de se demander si les trois femmes du film ne sont pas les fragments d'une même femme, éclatée. Andréane, la cinéaste, alimente la chanteuse, son personnage, avec les traits d'Estelle. Un moment, cette dernière proteste contre les artistes, grands parasites de la vie des autres: "Les créateurs sont des voleurs. Ma vie est à moi." En parasitant la "réalité", telle une "apprentie sorcière", la cinéaste est débordée par sa créature, influencée de plus en plus par le destin de la mélancolique Estelle. 
Sans contours définitoires nets, les deux femmes incarnent selon nous les deux pôles traditionnels de la mélancolie dont il a été question au début: le pôle créateur, figuré par Andréane, la cinéaste qui se jette tête perdue dans la production d'un film; le pôle dépressif, représenté par Estelle, la mélancolique, qui baisse les bras comme la figure de la Mélancolie dans la célèbre gravure de Dürer.

\section{II}

«Imaginer la mer, de nombreuses plages se perdant à l'infini sous un soleil printanier. " En bonne mélancolique, la femme de l'hôtel "pense» à la mer sans jamais pouvoir se décider à y aller. Dans le premier film de François Girard (venu instantanément à la célébrité internationale grâce à 32 Short Films about Glenn Gould, 1993), Cargo, la mer devient le "personnage" principal. Elle y incarne une force cosmique qui, lorsqu'elle se déchaîne, détruit tout projet humain.

Alice (Geneviève Rioux) emmène en bateau son père Philippe (Michel Dumont), bourreau de travail, en ce qui paraît être un petit voyage en mer. Séduction évidente qui suit le schème du complexe d'CEdipe. Le père, hésitant, se laisse finalement embarquer par sa fille. Certes, sur le voilier, il y a un autre homme qui aurait pu facilement devenir un rival. Or c'est un "sage» qui débite des vérités de Lapalice, sur l'impossibilité des voyages aujourd'hui, thème passablement éculé depuis Les Tristes Tropiques de ClaudeLévi Strauss: "Il n'y a plus rien à découvrir [...] Ailleurs, c'est ton dedans $[. .$.$] ce que vous cherchez au fond, vous le fuyez."$

On aura compris qu'il s'agit d'un voyage initiatique. On sait que chaque initiation comporte une épreuve. C'est le sens de l'ouragan que le voilier et son équipage essuient, perdant complètement le contrôle. Depuis l'antiquité grecque, le gouvernail du bateau est devenu la métaphore de la maîtrise rationnelle de l'Homme sur le monde, notamment du gouvernement de l'État. L'image est particulièrement prévalente dans CEdipe Roi de Sophocle, où l'on assiste également à un effondrement du logos dans l'ouragan de la libido d'Edipe.

Il va sans dire que, dans la foulée de la violence cataclysmique, tous les projets de travail établis par le patron Philippe 
s'écroulent complètement, à commencer évidemment par la "planification" de la journée: «Il faut que je rentre. J'ai planifié ma journée.»

Après la tempête, Philippe se trouve seul sur le bateau aux mâts brisés. Il est sauvé par un cargo. Vaisseau fantôme moderne (ou postmoderne) qui sort des brumes. Ses moteurs font un bruit infernal. "Bateau ivre" au cours incertain, puisque le capitaine a quitté le gouvernail - symbole, nous l'avons vu, de la maîtrise du monde - , battant selon la formule consacrée "pavillon de complaisance». Son équipage est constitué de "néo-Québécois", comme on appelle au Québec les immigrants récents qui ne sont pas "de souche". Le capitaine (Nelson Villagra) et l'officier (Manuel Aranguiz) se promènent nonchalamment sur le bateau, guidé par le pilote automatique. Ils trahissent leur origine par leur français aux lourds "accents». Dans les salles de machines sue un Lumpenproletariat sous-humain qui fait les jobs ingrats dont les Québécois de souche ne veulent pas. Plurisémique, le cargo signifie aussi, entre autres, le Québec ghettoïsé, incapable de concevoir - autrement que dans des discours lénifiants - une politique d'accueil de l'Autre.

N'empêche que c'est le cargo qui sauve le père. Désespéré, ce dernier veut retrouver sa fille. "Je veux voir ma fille." Il la voit dans une vision proche du délire qui la montre toujours vivante. Métissé entre deuil et mélancolie, Philippe combat, dénie ainsi, avec sa mélancolie, la mort de sa fille. Nous avons vu que pour le mélancolique, "seul" l'amour de la personne aimée est mort. Or, lorsque le cadavre de sa fille est déposé par la mer sur la plage, le déni de la mort de sa fille n'est plus possible. Du coup, les doutes du spectateur sur la nature du cargo se confirment sans jamais se résoudre. Nous restons avec des questions: le cargo a-t-il un statut réel, onirique ou hallucinatoire? Questions qui prouvent la grande ambivalence de cette métaphore du " cargo" qui porte littéralement sur d'autres rives incertaines sa cargaison humaine...

Le film se termine sur une image saisissante, hallucinatoire du père qui voit sa fille devenue mer (Mère?), sirène ensorcelante qui lui fait signe pour qu'il s'unisse, se fusionne avec elle dans les profondeurs de la mer abyssale. 
Venu cinq ans après Un zoo la nuit (1989), Léolo, le dernier film de Jean-Claude Lauzon, a été très mal reçu au Québec. Pour être franc, il n'a pas été reçu. Saisi par la "loi du silence», il a été frappé d'inexistence comme tout ce qui risque de déranger l'image "propre" - dans les deux sens du terme - que le Québec veut donner de lui-même.

Il n'est pas étonnant que plus d'un Québécois s'est détourné avec horreur de l'image dégradée, "déprimante", projetée de lui dans ce film. Il s'agit ici, en fait, de l'autre versant de la mélancolie au sens freudien: la régression narcissique et sadico-anale.

Commençons par la première. En effet, en s'appuyant sur Otto Rank, Freud n'a pas manqué de relever les affections narcissiques de la mélancolie. Le choix d'objet «[...] s'est produit sur une base narcissique, de sorte que l'investissement d'objet, si des difficultés s'élèvent contre lui, puisse régresser jusqu'au narcissisme" (p. 158). La régression narcissique de Léolo se manifeste par le fait qu'il ne voit dans l'Autre qu'une simple projection de sa libido, essentiellement auto-érotique. Léolo projette les fantasmes d'un Canadien français / Québécois qui refuse d'accepter sa "réalité » de Québécois «de souche». En rejetant son ancienne identité, il s'en construit une nouvelle, fantasmatique, en s'imaginant une généalogie italienne. "Tout le monde croit que je suis Canadien français. Lorsque moi je rêve, moi je ne suis pas."

Notons que les fantasmes de Léolo suivent tout à fait les schèmes du "roman familial" freudien (Famlienroman) dont nous avons analysé le fonctionnement dans l'histoire et dans le cinéma québécois ${ }^{8}$. Rappelons que le "roman familial" rabaisse, dénigre la famille biologique au profit d'une famille adoptive imaginaire, fortement idéalisée. Dans Léolo, la dépréciation de la famille biologique s'opère par un grand nombre de procédés dont nous énumérerons quelques-uns: la pauvreté; la cohabitation des humains avec les animaux (une dinde élit domicile dans la baignoire) qui fait dangereusement glisser l'humain sur la pente de l'animalité; la "production fécale", qui devient l'activité principale de la famille et, par voie de conséquence, les toilettes et la salle de bains, le centre du logement de la famille; Léolo qui note, à propos de son père: "La merde le rendait fier 


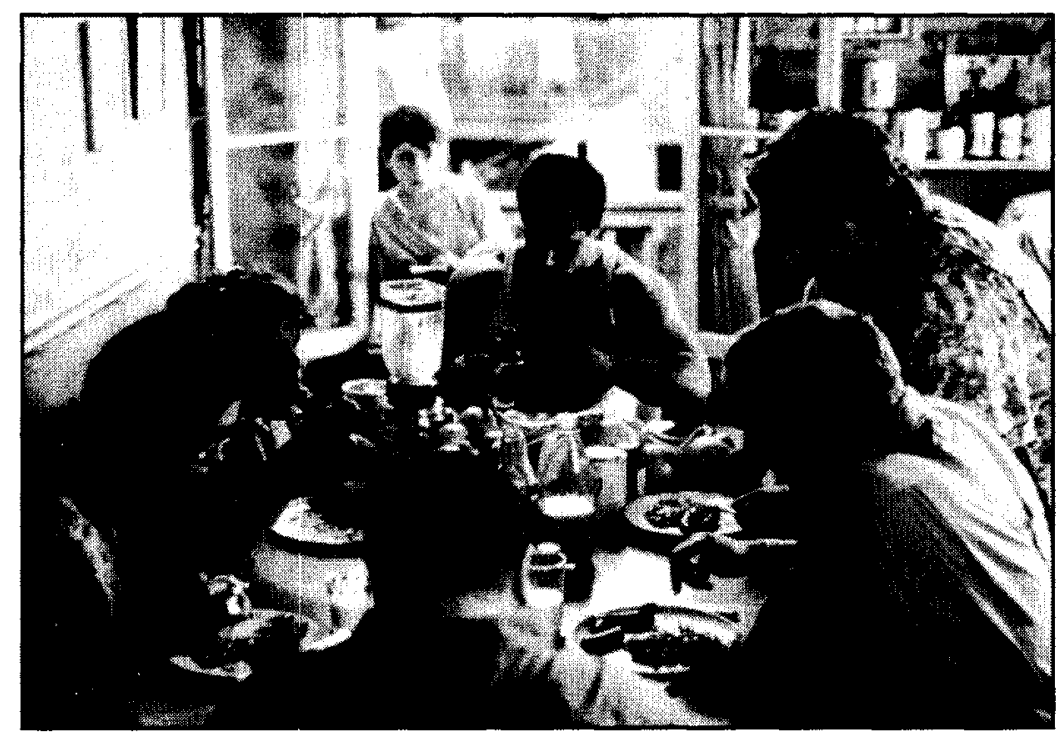

\section{Léolo de Jean-Claude Lauxon (1 992)}

Collection Cinémathèque québécoise

comme si je revenais de la guerre"; le père, un gros animal de trait, qui "bouffe» au sens premier, au point d'éclater, et qui ne cache pas son plaisir de mesurer la vaillance de son fils par la dimension de ses étrons ${ }^{9}$; la devise de cette famille, exprimée en termes cartésiens: "Je pense et je chie, donc je suis" et "Fais comme moi", dit la mère (Ginette Reno), assise sur le "trône", au jeune Léolo collé sur un pot de chambre.

Cette dégradation de la famille biologique va de pair avec une idéalisation excessive de la "famille" d'adoption: l'Italie. Au cas où on ne l'aurait pas compris, Léolo rejette vigoureusement la généalogie avec son père biologique: "Mais moi, je sais que je ne suis pas son fils. "Sa beauté fait de l'Italie le pays d'adoption par excellence: "L'Italie, c'est trop beau pour n'appartenir qu'aux Italiens. "

A côté du narcissisme, Léolo montre des poussées fortement sadico-anales, un des traits, selon Freud, de la mélancolie: "Quant à l'un des caractères frappants de la mélancolie, l'importance de l'angoisse d'appauvrissement, nous sommes tentés de la faire dériver de l'érotisme anal qui serait ici arraché à ses connexions et transformé par régression» (Freud, p. 163). 
S'expliquent ainsi les pulsions sadiques de Léolo: tortures du chat, tentative de meurtre de Léolo par le grand-père, assassinat du grand-père par Léolo à l'aide d'une machine sadique qui vise à prolonger les souffrances de la victime.

Fixée à l'oralité et à l'analité (on bouffe et on chie), la libido génitale, lorsqu'elle se manifeste, dévie vers un voyeurisme masturbatoire, marque d'un auto-érotisme narcissique qui n'ose pas faire le pas vers l'Autre. De là les peepshows masturbatoires entre la "chaste" Bianca et le grand-père, le tout observé par Léolo à partir d'une lucarne du plafond. Notons la grande fragilité de la génitalité, fût-elle perverse, qui a tendance à se résorber en oralité. Voir, à ce propos, les avatars d'un morceau de foie de porc: "support" masturbatoire de Léolo, rempli de sperme qui se transforme en plat cuisiné. Rappelons qu'oralité et onanisme président à la naissance de Léolo. Les avatars d'une tomate "importée" d'Italie sont aussi passionnants mais inversés par rapport au morceau de foie: la tomate, généreusement aspergée de sperme par un paysan sicilien, finit par tomber dans le sexe accueillant d'une Canadienne française.

Le père étant rabaissé à la quasi-animalité, c'est le grand-père qui tient son rôle dans Léolo. La rivalité œedipienne entre Léolo et le grand-père s'exacerbe autour du même objet d'amour, Bianca. La machine infernale, conçue par Léolo, punit son grand-père par où il a péché.

Pur fantasme ${ }^{10}$, Léolo met en scène le clivage, la "schize" entre fantasme, rêve et réalité. Clivage infranchissable par la plupart des frères et sœurs de Léolo. Cas schizophrènes lourds, ils passent le plus clair de leur temps en asile psychiatrique. Ce clivage entre réalité et fantasme est très bien illustré par le couple de frères Léolo et Fernand. Comme dans La Femme de l'hôtel, l'un et l'autre incarnent les deux pôles de la mélancolie: pôle créateur et pôle autodestructeur.

Mais éveillée par la lecture de L'Avalée des avalées, la «fantaisie" (la fantasie freudienne), l'imagination de Léolo se dégradent vite en ruminations mélancoliques, puisqu'elles ne débouchent pas sur une quelconque "réalisation". Car le "principe de réalité" est incarné par un autre personnage, Fernand, confirmant encore le profond clivage de l'univers de la famille de Léolo. 
Avec sa démarche lourde, Fernand représente la corporéité pure, dépourvue d'esprit, d'intelligence. Paquet de muscles, gonflés à l'aide de stéroïdes anabolisants, Fernand se dégonfle vite devant un chétif provocateur anglophone: il tremble, il en a peur. Pur corps, Fernand s'écrase littéralement devant l'adversité, il a vitalement besoin de Léolo, son complément pensant, imaginant, son complément d'esprit.

Imagination pure, Léolo a besoin aussi d'un complément de réalité que Fernand, trop avachi, ne pourra assumer. De là la présence d'un "dompteur de rêves" (Pierre Bourgault) qui fouille littéralement les poubelles de Léolo, en quête de ces "déchets" imaginaires que Léolo n'arrive pas à assembler en œuvre. À l'image de la Melencolia I de Dürer, Léolo, entouré d'«instruments» inutiles, est plongé dans une rêverie profonde dont personne ne peut l'arracher.

En conclusion, nous avons constaté que les trois films québécois s'inscrivent dans la mouvance du syndrome postréférendaire (1980), période où l'âme québécoise a traversé une crise profonde d'incertitudes, de doutes et d'inquiétudes. Nous y avons noté une quête exacerbée d'un objet d'amour, d'un idéal hors d'atteinte. Jamais déclaré mort, cet idéal vacille au loin comme Saturne, planète de la mélancolie. Couvert de l'ombre mélancolique, le sujet, dans l'espoir d'attirer l'attention, la pitié de l'objet d'amour, se rabaisse, se déprécie, se laissant gagner par un "délire de petitesse" (Freud).

Dans La Femme de l'hôtel, la dépréciation est vécue comme une dépression extrême d'Estelle, incapable d'aimer l'Autre (Andréane) parce que, fondamentalement, elle ne s'aime pas. Dans Cargo, Philippe, un patron qui a "géré" sa vie jusque-là avec beaucoup de superbe, est arraché par une tempête en mer à son "trône d'assurance" (Edgar Morin). Naufragé, en haillons sur son voilier en dérive, il a perdu l'orientation de sa vie. Sauvé par le cargo, l'existence de Philippe ressemble dorénavant de plus en plus à celle de son équipage exploité, soumis à une condition d'esclaves. C'est sans doute dans Léolo que la dépréciation du sujet atteint son sommet: l'humain y cohabite avec l'animalité la plus basse (insectes et rats), son activité principale étant la production de la matière fécale. Le fort 
investissement de l'érotisme anal fait naître de puissantes pulsions sadiques.

Doutant, après l'échec du référendum de 1980 , de sa capacité d' “indépendance" (Freud), le sujet québécois traverse une période de profonde auto-dépréciation. En parfait mélancolique, il s'inflige à lui-même des souffrances, payant en quelque sorte de sa personne pour l'éloignement, la disparition de l'objet aimé.

\section{Collège de Rosemont}

\section{NOTES}

1 Voir à ce propos notre texte, "Naissance de la psychanalyse dans la métaphore ou les deux regards de Freud", à paraitre dans un collectif publié à l'automne à L'Harmattan intitulé Transports de psychanlayse.

2 Pharmakon, notion dégagée par Derrida dans sa lecture du Phèdre de Platon, "La pharmacie de Platon ", rééditée en appendice au Phèdre (Paris: Garnier-Flammarion, 1989 , p. $255-405$ ). Voir aussi notre texte: «La petite Aurore : la pharmacie mortelle d'un Québec naissant ", dans Cinéma de l'imaginaire québécois (Montréal: l'Hexagone, 1990, p. 27-50).

3 Voir à ce sujet "Comment le paysan français devient "habitant" ", Du Canada au Québec. Généalogie d'une histoire (Montréal : l'Hexagone, 1987, p. 237-245).

4 Pour plus de détails, "La contagion nomade», Du Canada au Québec. Généalogie d'une histoire (Montréal: Hexagone, 1987, p. 211-214).

5 Je renvoie à nouveau au texte "La contagion nomade", Du Canada au Québec. Généalogie d'une histoire (Montréal: l'Hexagone, 1987, p. 211-214).

6 La langue anglaise fait une différence entre voyage qui implique un but, une destination et travel, comme dans Gulliver's Travels de Swift, des randonnées (de même souche que l'anglais at random, au hasard), des errances faites au hasard.

7 En allemand le mot irren renvoie à trois champs sémantiques, signifiant comme en français errer (aller çà et là), se tromper, et être fou, le fou étant le Irre.

8 Voir «Le roman familial canadien", Du Canada au Québec. Généalogie d'une histoire (Montréal : l'Hexagone, 1987, p. 289-308).

9 Voir là-dessus le texte de Freud, "Caractère et érotisme anal ", Névrose, psychose et perversion (Paris: PUF, 1974, p. 143-148).

10 Voir le texte de Freud, "Le créateur littéraire et la fantaisie", LInquiétante Étrangeté et autres essais (Paris: Gallimard, 1985, p. 29-46).

\section{OUVRAGES CITÉS}

Aristote. Problème XXX. Paris: Rivages, 1988.

Freud, Sigmund. “Deuil et mélancolie», Métapsychologie. Paris: Gallimard (1968), p. 147-174.

Klibansky, Raymond, Erwin Panofsky et Fritz Saxl. Saturne et la Mélancolie. Paris : Gallimard, 1989. 